\title{
Radon concentration measurements in surface water samples from Van Lake, Turkey using CR-39 detectors
}

\author{
Halime Kayaköküa,* (D), Mahmut Doğrub (D) \\ a Bitlis Eren University, Department of Physics, TR-13000, Bitlis Turkey \\ b Fırat University, Department of Physics, TR-23169, Elazığ Turkey
}

\section{ART I C LE INFO}

\section{Article history:}

Received 24 March 2020

Received in revised form 24 March 2020

Accepted 04 June 2020

\section{Keywords:}

Radon,

Van Lake,

CR-39 detector,

RadoSYS

\begin{abstract}
A B S T R A C T
In this study, radon concentration was examined in surface water samples taken from Van Lake in spring and autumn. The samples were taken along the coastline from Tatvan, where the active fault line lies, until Erciş. Analyses were performed by using CR-39 solid nuclear track detectors and RadoSYS radon measurement system. Radon measurement parameters $\left(\mathrm{C}_{\mathrm{Rn}}, \mathrm{E}_{\mathrm{s}}, \mathrm{E}_{\mathrm{m}}\right.$ and $\left.\mathrm{EC}_{\mathrm{Ra}}\right)$ were calculated with the data obtained. The results of this study were compared with the limit values given for radon in water and the results obtained in similar studies.
\end{abstract}

(C) 2020. Turkish Journal Park Academic. All rights reserved.

\section{Introduction}

Radon is a noble gas with a half-life of 3.82 days and it occurs as a result of the radioactive conversion of $226 \mathrm{Ra}$ (Radium) located in the decay chain of $238 \mathrm{U}$ (Uranium). Radioactive radon gas, which is colorless, odorless, tasteless and short-lived decay products in the atmosphere account for about $50 \%$ of the radiation that people are exposed to from natural sources (UNSCEAR, 2000). ${ }^{222} \mathrm{Rn}$, which is approximately seven times heavier than air, is collected in hollows and dissolved in water (Alkan and Göksel, 1975). It is not possible to find water in its pure form in the natural environment. Radiation pollutes water and threatens human health. The contamination of water by radiation results from the natural radioisotopes in the atmosphere and soil, reactor accidents, nuclear weapon tests and medical radioactive wastes (Yaramıș, 1985). Besides the solubility of radon gas in water there is also a tendency to escape into the environment where the dissolved radon is located. As a result, high radon concentrations in groundwater and thermal waters, it poses a great danger not only for people who drink water, but also for people who breathe it (UNSCEAR, 2000).

One of the significant factors that need to be determined in terms of water usability is the level of radioactivity. For this reason, the conformity of drinking and domestic water to standards should be investigated with analyses on the radioisotopes available in water (Varol, 2011). The natural radioactivity of the water arises from the radioactive masses or minerals, which they pass through or are in contact with.

Radon concentration in water changes depending on some factors such as the emission of radon from the rocks that the water contacts, temperature, pressure, precipitation and seismic activities (Ilani et al., 2006; Sannappa et al., 2006). Some rock types contain uranium in concentrations higher than $5 \mathrm{ppm}$. These are granites, syenites, pegmatites, acid volcanic rocks and gneisses (Shashikumar et al., 2009; UNSCEAR, 2000).

Radon measurement techniques divide into two groups as active and passive measurement techniques. In this study, the

* Corresponding author.

Tel.: +90 434 222 0000; fax: +90 4342220101

E-mail address: hkayakoku@beu.edu.tr

ORCID : 0000-0003-4036-4012 (Halime KAYAKÖKÜ), 0000-0002-0015-0629 (Mahmut DOĞRU) 
passive measurement technique was employed to detect radon gas concentrations in water. CR-39 solid track detectors are frequently used in passive measurement technique (Kayakökü et al., 2016; Turhan et al., 2018; Büyükuslu et al., 2018; Alali et al., 2019).

The aim of present research study was to determine the radon concentration, effective radium content, the surface and mass diffusion rates of radon in surface water samples taken from Van Lake in spring and autumn. For this purpose, CR-39 solid nuclear track detectors were used. Radon analyzes were done using RadoSYS system.

\section{Material and Method}

\subsection{Research area}

Van Lake is located in the east of Turkey and the GPS coordinates of its geographical location are $38^{\circ} 38^{\prime} 27^{\prime \prime}$ north and $42^{\circ} 48^{\prime} 45^{\prime \prime}$ east. It was formed as a result of the explosion of the Nemrut volcanic mountain, which is located within the borders of the province of Bitlis. Van Lake is a volcanic embankment with a surface area of $3790 \mathrm{~km}^{2}$ as an enclosed lake. The water of this lake is an alkaline lake with a salinity rate of $0.19 \%$ and $\mathrm{pH}$ of 9.8 on average.

Water samples were collected using a Hydro bios ruttner water sampler from 24 stations within Van Lake. $15 \mathrm{ml}$ of $\mathrm{HNO}_{3}$ (nitric acid) was added to each 1 liter of sample. (Bohus-Saja et al., 1997). In this way, $\mathrm{pH} \leq 2$ was obtained for water samples, and it was ensured that the elements in the samples would not precipitate and stick on the surface of the sample container (Bohus-Saja et al., 1997).
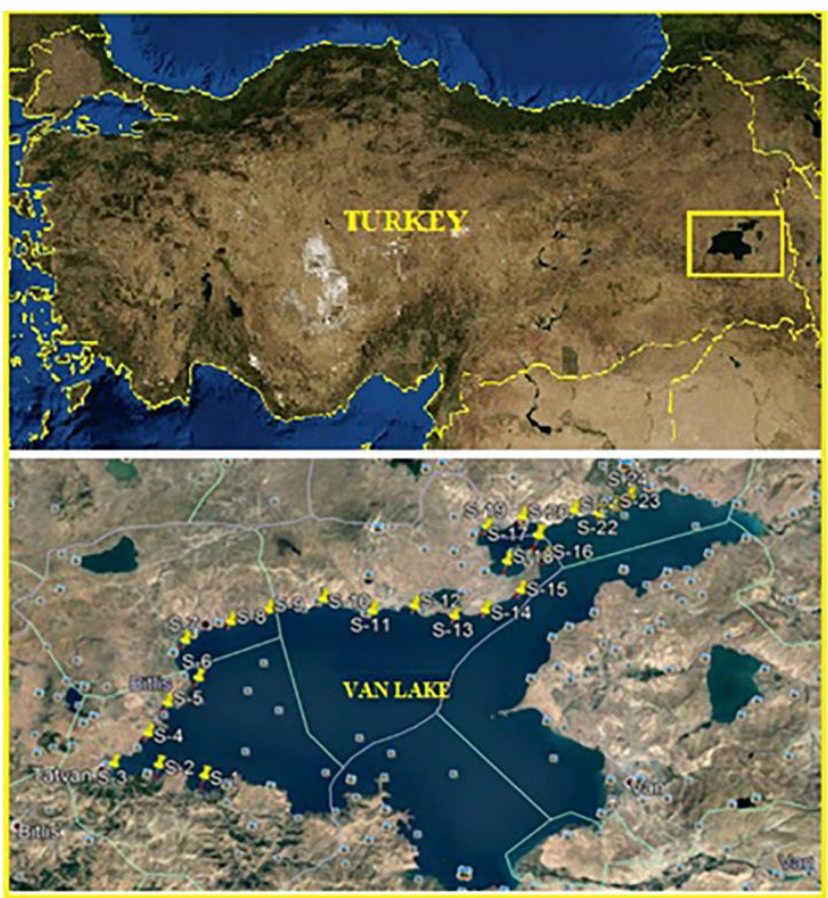

Figure 1. Research area and sampling points
In present study, the radon concentrations, exhalation rates and radium contents were calculated using CR-39 detectors for forty eight surface water samples. After the water samples were placed in the sample containers, they were left for a month to ensure radioactive equilibrium. CR-39 detectors were prepared in pieces of $1 \mathrm{~cm} \times 1 \mathrm{~cm}$ dimensions. After a onemonth waiting period, CR-39 detectors were placed in sample containers. Likewise, after a one-month waiting period, the detectors were removed from the containers.

The cylindrical polyethylene sample containers, in which the sample and the CR-39 detector are placed, are shown in Figure 2.

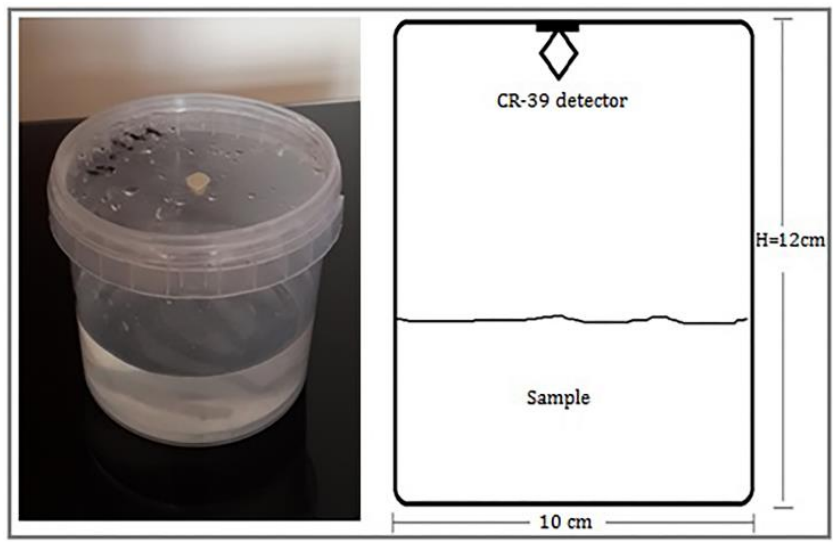

Figure 2. The structure cylindrical polyethylene sample container used for radon measurements

After the one-month waiting period was over, the sample containers were opened, and the detectors were removed from the containers. During this time, the chemical etching process was employed in order to make the traces of the alpha particles left on the detectors apparent. For this purpose, a $30 \% \mathrm{NaOH}$ solution was prepared and distributed into the beakers equally. After the detectors were placed in the solution, the beakers were kept in the oven at $60{ }^{\circ} \mathrm{C}$ for 18 hours. Afterwards, the detectors were removed from the beakers which were taken out of the oven and then washed with pure water. Following the completion of the washing process, the detectors were kept aside for about 1 hour to dry. After drying up, the detectors were ready for the tracking procedure.

Tracks of alpha particles hitting Cr-39 detectors during the waiting period were shown in Figure 3.

Radon analysis operations were carried out using the Passive Radon Detector System (RadoSYS system) in Bitlis Eren University Nuclear Physics Research Laboratory. The radon tracking system (Figure 3) consists of a $500 \times$ magnification microscope and software connected to the computer. The program works with the LINUX operating system. First, CR-39 detectors were placed on the slides. After the detectors on the slides were divided into 144 equal parts by the optical tracking system, the traces on the detectors were counted. After the periods of keeping the detectors in the dishes containing the 
samples were entered on the program, radon density and radon activity concentration were separately calculated by the program for each sample.

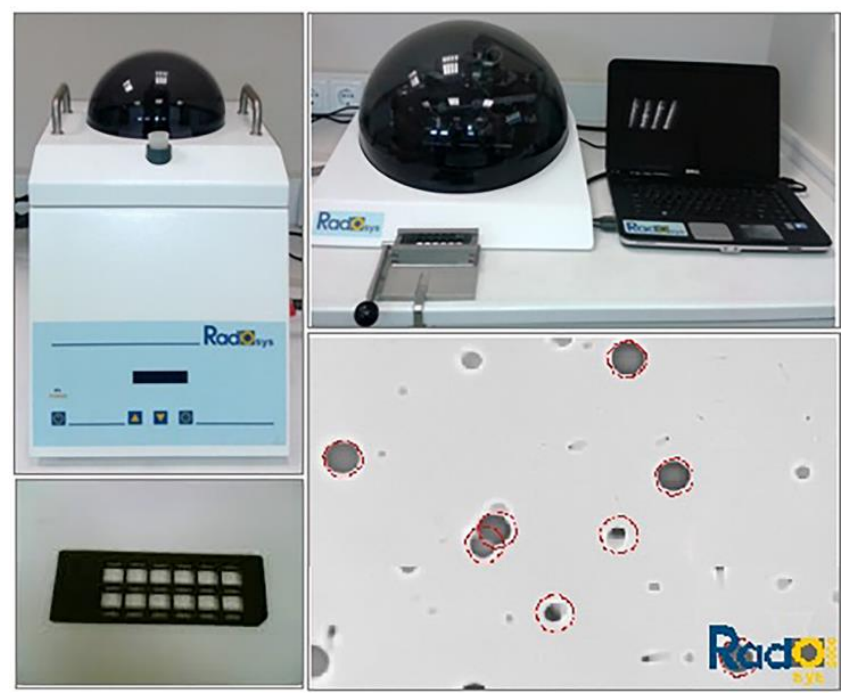

Figure 3. Radon track reading system and the tracks of alpha particles in the CR-39 detector

In Figure 3, the tracks of alpha particles hitting the CR-39 detector can be seen. While calculating radon concentrations, it is necessary to detect the background value of the detector placed in the dish when there is no sample. For this, the detectors in the sample dishes and the detector in the empty dish were tracked on the detector tracking system in the same way, and the net track densities were acquired by the subtraction of the track densities from each other.

\subsection{Radon concentration calculations}

After specifying the net trace densities formed on the CR-39 detectors, the radon concentrations were calculated by Equation (1) using the obtained net trace densities, the periods the detectors were kept in the sample containers and the calibration factor.

$C_{R n}=\frac{\rho \times f}{\Delta t}$

where $C_{R n}$ is radon concentration $\left(\mathrm{Bq} / \mathrm{m}^{3}\right), \rho$ is net track density (track $/ \mathrm{mm}^{2}$ ), $f$ is calibration factor and $\Delta t$ is detector exposure time (in hour) (Tokonami et al., 2005). Calibration factor for CR-39 solid nuclear trace detectors is 44.47 $\left(\mathrm{kBqh} / \mathrm{m}^{3}\right) /\left(\right.$ track $\left./ \mathrm{mm}^{2}\right)$.

The equation used to calculate the effective radium content $\left(E_{\mathrm{Ra}}\right)$ was shown in Equation 2.

$$
E C_{R a}=\frac{\rho \times h \times A \times f}{V \times T_{e f f}}
$$

\subsection{Effective radium content $\left(E C_{R a}\right)$}

where $h$ is distance between detector and sample (m), $A$ is the surface area of sample container $\left(\mathrm{m}^{2}\right), T$ is total exposure time (h), $T_{\text {eff }}$ is effective exposure time $\left[\mathrm{T}_{\text {eff }}=\mathrm{T}+1 / \lambda\left(\mathrm{e}^{\lambda \mathrm{T}}-1\right)\right], V$ is sample volume $\left(\mathrm{m}^{3}\right)$ (Jönsson et al., 1999).

\subsection{Radon surface and mass exhalation rates $\left(E_{s}, E_{m}\right)$}

Radon surface exhalation rates $\left(E_{s}\right)$ and radon mass exhalation rates $\left(E_{m}\right)$ were calculated using Equation (3) and Equation (4), respectively.

$$
\begin{aligned}
& E_{S}=\frac{\rho \times f \times h \times \lambda}{T_{e f f}} \\
& E_{m}=E_{S} \times \frac{A}{M}
\end{aligned}
$$

where, $\lambda\left(2.1 \times 10^{-6}\right)$ is ${ }^{222} \mathrm{Rn}$ decay constant, $h$ is height of the sample container (m), $A$ is surface area of the sample in the container $\left(\mathrm{m}^{2}\right)$ and $M$ is the mass of the sample in the sample container $(\mathrm{kg})$.

\section{Results and discussion}

Radon concentrations $\left(\mathrm{C}_{\mathrm{Rn}}\right)$, radon surface exhalation rates $\left(E_{s}\right)$, radon mass exhalation rates $\left(E_{m}\right)$ and effective radium contents $\left(\mathrm{EC}_{\mathrm{Ra}}\right)$ for surface water samples taken in spring and autumn periods were given in Table 1 and Table 2, respectively. While the comparison of the radon concentration values in the surface water samples of the spring and autumn period with the limit value recommended by USEPA (United States Environmental Protection Agency, 2012) was shown in Figure 4 and Figure 5, respectively, the seasonal comparison of the radon concentration values was shown in Figure 6.

$$
{ }^{\mathrm{EC}} \mathrm{Ra}^{=} \frac{}{V \times T_{\text {eff }}}
$$


Table 1. The $C_{R n}, E_{s}, E_{m}$ and $E_{R a}$ values of surface water sample in spring period (Kayakökü and Doğru, 2019).

\begin{tabular}{|c|c|c|c|c|c|c|}
\hline \multirow{2}{*}{$\begin{array}{c}\text { Sample } \\
\text { ID }\end{array}$} & \multicolumn{2}{|c|}{ Sample locations } & \multirow{2}{*}{$\begin{array}{c}\mathrm{C}_{\mathrm{Rn}} \\
\left(\mathrm{Bq} / \mathrm{m}^{3}\right)\end{array}$} & \multirow{2}{*}{$\begin{array}{c}E_{s} \\
\left(B q / m^{2} \cdot h\right) \times 10^{-1}\end{array}$} & \multirow{2}{*}{$\begin{array}{c}E_{m} \\
\left(B q / m^{3} \cdot h\right) \times 10^{-3}\end{array}$} & \multirow{2}{*}{$\begin{array}{c}\mathrm{EC}_{\mathrm{Ra}} \\
\left(\mathrm{Bq} / \mathrm{m}^{3}\right)\end{array}$} \\
\hline & Latitude & Longitude & & & & \\
\hline Y.S-1 & $38^{\circ} 29^{\prime} 04.3^{\prime \prime}$ & $42^{\circ} 29^{\prime} 22.5^{\prime \prime}$ & $10.00 \pm 0.92$ & $0.12 \pm 0.01$ & $0.14 \pm 0.01$ & $0.08 \pm 0.01$ \\
\hline Y.S-2 & $38^{\circ} 30^{\prime} 02.8^{\prime \prime}$ & $42^{\circ} 23^{\prime} 39.8^{\prime \prime}$ & $18.30 \pm 1.69$ & $0.14 \pm 0.01$ & $0.21 \pm 0.02$ & $0.15 \pm 0.01$ \\
\hline Y.S-3 & $38^{\circ} 30^{\prime} 03.1^{\prime \prime}$ & $42^{\circ} 18^{\prime} 03.4^{\prime \prime}$ & $43.41 \pm 4.00$ & $0.32 \pm 0.03$ & $1.31 \pm 0.12$ & $0.72 \pm 0.07$ \\
\hline Y.S-4 & $38^{\circ} 33^{\prime} 17.7^{\prime \prime}$ & $42^{\circ} 22^{\prime} 11.4^{\prime \prime}$ & $78.60 \pm 7.25$ & $0.58 \pm 0.05$ & $2.15 \pm 0.20$ & $1.30 \pm 0.12$ \\
\hline Y.S-5 & $38^{\circ} 36^{\prime} 26.5^{\prime \prime}$ & $42^{\circ} 24^{\prime} 30.9^{\prime \prime}$ & $33.85 \pm 3.12$ & $0.25 \pm 0.02$ & $1.02 \pm 0.09$ & $0.56 \pm 0.05$ \\
\hline Y.S-6 & $38^{\circ} 39^{\prime} 05.3^{\prime \prime}$ & $42^{\circ} 28^{\prime} 14.7^{\prime \prime}$ & $50.00 \pm 4.61$ & $0.37 \pm 0.03$ & $0.48 \pm 0.04$ & $0.35 \pm 0.03$ \\
\hline Y.S-7 & $38^{\circ} 42^{\prime} 56.9^{\prime \prime}$ & $42^{\circ} 26^{\prime} 29.8^{\prime \prime}$ & $31.07 \pm 2.8$ & $0.23 \pm 0.02$ & $0.94 \pm 0.09$ & $0.51 \pm 0.05$ \\
\hline Y.S-8 & $38^{\circ} 44^{\prime} 55.7^{\prime \prime}$ & $42^{\circ} 32^{\prime} 03.1^{\prime \prime}$ & $45.20 \pm 4.17$ & $0.33 \pm 0.03$ & $0.44 \pm 0.04$ & $0.31 \pm 0.03$ \\
\hline Y.S-9 & $38^{\circ} 46^{\prime} 15.3^{\prime \prime}$ & $42^{\circ} 36^{\prime} 44.6^{\prime \prime}$ & $10.75 \pm 0.99$ & $0.08 \pm 0.01$ & $0.33 \pm 0.03$ & $0.18 \pm 0.02$ \\
\hline Y.S-10 & $38^{\circ} 47^{\prime} 31.5^{\prime \prime}$ & $42^{\circ} 43^{\prime} 30.5^{\prime \prime}$ & $32.00 \pm 2.95$ & $0.24 \pm 0.02$ & $0.31 \pm 0.03$ & $0.22 \pm 0.02$ \\
\hline Y.S-11 & $38^{\circ} 46^{\prime} 22.4^{\prime \prime}$ & $42^{\circ} 49^{\prime} 41.6^{\prime \prime}$ & $80.85 \pm 7.45$ & $0.60 \pm 0.06$ & $2.30 \pm 0.21$ & $1.34 \pm 0.12$ \\
\hline Y.S-12 & $38^{\circ} 47^{\prime} 00.4^{\prime \prime}$ & $42^{\circ} 54^{\prime} 52.7^{\prime \prime}$ & $16.24 \pm 1.50$ & $0.12 \pm 0.01$ & $0.16 \pm 0.01$ & $0.11 \pm 0.01$ \\
\hline Y.S-13 & $38^{\circ} 46^{\prime} 01.1^{\prime \prime}$ & $42^{\circ} 59^{\prime} 53.7^{\prime}$ & $41.82 \pm 3.86$ & $0.31 \pm 0.03$ & $0.48 \pm 0.04$ & $0.35 \pm 0.03$ \\
\hline Y.S-14 & $38^{\circ} 46^{\prime} 43.2^{\prime \prime}$ & $43^{\circ} 03^{\prime} 41.9^{\prime \prime}$ & $58.24 \pm 5.37$ & $0.43 \pm 0.04$ & $0.56 \pm 0.05$ & $0.41 \pm 0.04$ \\
\hline Y.S-15 & $38^{\circ} 48^{\prime} 47.6^{\prime \prime}$ & $43^{\circ} 08^{\prime} 04.7^{\prime \prime}$ & $49.29 \pm 4.54$ & $0.33 \pm 0.03$ & $1.35 \pm 0.12$ & $0.73 \pm 0.07$ \\
\hline Y.S-16 & $38^{\circ} 52^{\prime} 19.1^{\prime \prime}$ & $43^{\circ} 11^{\prime} 12.9^{\prime \prime}$ & $62.80 \pm 5.79$ & $0.37 \pm 0.03$ & $0.66 \pm 0.06$ & $0.47 \pm 0.04$ \\
\hline Y.S-17 & $38^{\circ} 54^{\prime} 24.9^{\prime \prime}$ & $43^{\circ} 10^{\prime} 04.9^{\prime \prime}$ & $56.24 \pm 5.19$ & $0.42 \pm 0.04$ & $0.54 \pm 0.05$ & $0.39 \pm 0.03$ \\
\hline Y.S-18 & $38^{\circ} 51^{\prime} 45.3^{\prime \prime}$ & $43^{\circ} 06^{\prime} 14.9^{\prime \prime}$ & $60.00 \pm 5.53$ & $0.44 \pm 0.04$ & $0.58 \pm 0.05$ & $0.42 \pm 0.04$ \\
\hline Y.S-19 & $38^{\circ} 55^{\prime} 16.7^{\prime \prime}$ & $43^{\circ} 03^{\prime} 42.0^{\prime \prime}$ & $25.53 \pm 2.35$ & $0.12 \pm 0.01$ & $0.49 \pm 0.04$ & $0.26 \pm 0.01$ \\
\hline Y.S-20 & $38^{\circ} 56^{\prime} 23.5^{\prime \prime}$ & $43^{\circ} 08^{\prime} 05.3^{\prime \prime}$ & 28.282 .61 & $0.21 \pm 0.03$ & $0.86 \pm 0.08$ & $0.47 \pm 0.03$ \\
\hline Y.S-21 & $38^{\circ} 57^{\prime} 08.6^{\prime \prime}$ & $43^{\circ} 14^{\prime} 30.1^{\prime \prime}$ & $18.65 \pm 1.72$ & $0.14 \pm 0.01$ & $0.18 \pm 0.02$ & $0.13 \pm 0.01$ \\
\hline Y.S-22 & $38^{\circ} 56^{\prime} 50.8^{\prime \prime}$ & $43^{\circ} 17^{\prime} 39.1^{\prime \prime}$ & $30.10 \pm 2.78$ & $0.22 \pm 0.02$ & $0.29 \pm 0.03$ & $0.21 \pm 0.02$ \\
\hline Y.S-23 & $38^{\circ} 57^{\prime} 41.5^{\prime \prime}$ & $43^{\circ} 19^{\prime} 27.5^{\prime \prime}$ & $68.45 \pm 6.31$ & $0.51 \pm 0.05$ & $2.07 \pm 0.19$ & $1.49 \pm 0.14$ \\
\hline Y.S-24 & $38^{\circ} 58^{\prime} 46.7^{\prime \prime}$ & $43^{\circ} 21^{\prime} 23.8^{\prime \prime}$ & $20.55 \pm 1.89$ & $0.15 \pm 0.01$ & $0.20 \pm 0.02$ & $0.14 \pm 0.01$ \\
\hline \multicolumn{3}{|c|}{ Average } & $40.43 \pm 3.73$ & $0.29 \pm 0.03$ & $0.75 \pm 0.07$ & $0.47 \pm 0.04$ \\
\hline \multicolumn{3}{|c|}{ Maximum } & $80.85 \pm 7.45$ & $0.60 \pm 0.06$ & $2.30 \pm 0.21$ & $1.49 \pm 0.14$ \\
\hline \multicolumn{3}{|c|}{ Minimum } & $10.00 \pm 0.92$ & $0.08 \pm 0.01$ & $0.14 \pm 0.01$ & $0.08 \pm 0.01$ \\
\hline
\end{tabular}


Table 2. The $C_{R n}, E_{s}, E_{m}$ and $E_{R a}$ values of surface water sample in autumn period (Kayakökü and Doğru, 2019).

\begin{tabular}{|c|c|c|c|c|c|c|}
\hline \multirow{2}{*}{$\begin{array}{l}\text { Sample } \\
\text { ID }\end{array}$} & \multicolumn{2}{|c|}{ Sample locations } & \multirow{2}{*}{$\begin{array}{c}\mathrm{C}_{\mathrm{Rn}} \\
\left(\mathrm{Bq} / \mathrm{m}^{3}\right)\end{array}$} & \multirow{2}{*}{$\begin{array}{c}E_{s} \\
\left(B q / m^{2} \cdot h\right) \times 10^{-1}\end{array}$} & \multirow{2}{*}{$\begin{array}{c}E_{m} \\
\left(B q / m^{3} \cdot h\right) \times 10^{-3}\end{array}$} & \multirow{2}{*}{$\begin{array}{c}\mathrm{EC}_{\mathrm{Ra}} \\
\left(\mathrm{Bq} / \mathrm{m}^{3}\right)\end{array}$} \\
\hline & Latitude & Longitude & & & & \\
\hline Y.S-1 & $38^{\circ} 29^{\prime} 04.3^{\prime \prime}$ & $42^{\circ} 29^{\prime} 22.5^{\prime \prime}$ & $\mathrm{a}$ & $\mathrm{a}$ & a & a \\
\hline Y.S-2 & $38^{\circ} 30^{\prime} 02.8^{\prime \prime}$ & $42^{\circ} 23^{\prime} 39.8^{\prime \prime}$ & a & a & a & a \\
\hline Y.S-3 & $38^{\circ} 30^{\prime} 03.1^{\prime \prime}$ & $42^{\circ} 18^{\prime} 03.4^{\prime \prime}$ & $48.59 \pm 4.48$ & $0.36 \pm 0.03$ & $1.47 \pm 0.14$ & $0.80 \pm 0.07$ \\
\hline Y.S-4 & $38^{\circ} 33^{\prime} 17.7^{\prime \prime}$ & $42^{\circ} 22^{\prime} 11.4^{\prime \prime}$ & $125.33 \pm 11.60$ & $1.15 \pm 0.11$ & $4.70 \pm 0.43$ & $2.57 \pm 0.24$ \\
\hline Y.S-5 & $38^{\circ} 36^{\prime} 26.5^{\prime \prime}$ & $42^{\circ} 24^{\prime} 30.9^{\prime \prime}$ & $28.76 \pm 2.65$ & $0.45 \pm 0.04$ & $0.66 \pm 0.06$ & $0.55 \pm 0.05$ \\
\hline Y.S-6 & $38^{\circ} 39^{\prime} 05.3^{\prime \prime}$ & $42^{\circ} 28^{\prime} 14.7^{\prime \prime}$ & $65.00 \pm 5.99$ & $0.48 \pm 0.04$ & $2.00 \pm 0.18$ & $1.18 \pm 0.11$ \\
\hline Y.S-7 & $38^{\circ} 42^{\prime} 56.9^{\prime \prime}$ & $42^{\circ} 26^{\prime} 29.8^{\prime \prime}$ & $86.43 \pm 7.97$ & $0.64 \pm 0.06$ & $2.61 \pm 0.24$ & $1.43 \pm 0.13$ \\
\hline Y.S-8 & $38^{\circ} 44^{\prime} 55.7^{\prime \prime}$ & $42^{\circ} 32^{\prime} 03.1^{\prime \prime}$ & $46.50 \pm 4.29$ & $0.34 \pm 0.03$ & $1.45 \pm 0.13$ & $0.84 \pm 0.08$ \\
\hline Y.S-9 & $38^{\circ} 46^{\prime} 15.3^{\prime \prime}$ & $42^{\circ} 36^{\prime} 44.6^{\prime \prime}$ & a & a & a & a \\
\hline Y.S-10 & $38^{\circ} 47^{\prime} 31.5^{\prime \prime}$ & $42^{\circ} 43^{\prime} 30.5^{\prime \prime}$ & $54.92 \pm 5.06$ & $0.47 \pm 0.04$ & $1.96 \pm 0.18$ & $1.08 \pm 0.10$ \\
\hline Y.S-11 & $38^{\circ} 46^{\prime} 22.4^{\prime \prime}$ & $42^{\circ} 49^{\prime} 41.6^{\prime \prime}$ & $171.17 \pm 15.80$ & $1.27 \pm 0.12$ & $5.19 \pm 0.48$ & $3.28 \pm 0.26$ \\
\hline Y.S-12 & $38^{\circ} 47^{\prime} 00.4^{\prime \prime}$ & $42^{\circ} 54^{\prime} 52.7^{\prime \prime}$ & $45.14 \pm 4.16$ & $0.32 \pm 0.03$ & $1.84 \pm 0.17$ & $1.10 \pm 0.10$ \\
\hline Y.S-13 & $38^{\circ} 46^{\prime} 01.1^{\prime \prime}$ & $42^{\circ} 59^{\prime} 53.7^{\prime}$ & $60.55 \pm 5.53$ & $0.45 \pm 0.04$ & $0.89 \pm 0.08$ & $1.06 \pm 0.10$ \\
\hline Y.S-14 & $38^{\circ} 46^{\prime} 43.2^{\prime \prime}$ & $43^{\circ} 03^{\prime} 41.9^{\prime \prime}$ & a & a & a & a \\
\hline Y.S-15 & $38^{\circ} 48^{\prime} 47.6^{\prime \prime}$ & $43^{\circ} 08^{\prime} 04.7^{\prime \prime}$ & a & a & a & a \\
\hline Y.S-16 & $38^{\circ} 52^{\prime} 19.1^{\prime \prime}$ & $43^{\circ} 11^{\prime} 12.9^{\prime \prime}$ & $44.21 \pm 4.08$ & $0.33 \pm 0.03$ & $1.36 \pm 0.13$ & $0.80 \pm 0.07$ \\
\hline Y.S-17 & $38^{\circ} 54^{\prime} 24.9^{\prime \prime}$ & $43^{\circ} 10^{\prime} 04.9^{\prime \prime}$ & a & a & a & a \\
\hline Y.S-18 & $38^{\circ} 51^{\prime} 45.3^{\prime \prime}$ & $43^{\circ} 06^{\prime} 14.9^{\prime \prime}$ & $60.00 \pm 5.53$ & $0.44 \pm 0.04$ & $1.85 \pm 0.17$ & $1.09 \pm 0.10$ \\
\hline Y.S-19 & $38^{\circ} 55^{\prime} 16.7^{\prime \prime}$ & $43^{\circ} 03^{\prime} 42.0^{\prime \prime}$ & $72.45 \pm 6.68$ & $0.53 \pm 0.05$ & $2.23 \pm 0.21$ & $1.32 \pm 0.12$ \\
\hline Y.S-20 & $38^{\circ} 56^{\prime} 23.5^{\prime \prime}$ & $43^{\circ} 08^{\prime} 05.3^{\prime \prime}$ & $28.28 \pm 2.61$ & $0.21 \pm 0.02$ & $0.38 \pm 0.04$ & $0.42 \pm 0.04$ \\
\hline Y.S-21 & $38^{\circ} 57^{\prime} 08.6^{\prime \prime}$ & $43^{\circ} 14^{\prime} 30.1^{\prime \prime}$ & $36.80 \pm 3.39$ & $0.27 \pm 0.02$ & $0.50 \pm 0.05$ & $0.58 \pm 0.05$ \\
\hline Y.S-22 & $38^{\circ} 56^{\prime} 50.8^{\prime \prime}$ & $43^{\circ} 17^{\prime} 39.1^{\prime \prime}$ & a & a & a & a \\
\hline Y.S-23 & $38^{\circ} 57^{\prime} 41.5^{\prime \prime}$ & $43^{\circ} 19^{\prime} 27.5^{\prime \prime}$ & a & a & a & a \\
\hline Y.S-24 & $38^{\circ} 58^{\prime} 46.7^{\prime \prime}$ & $43^{\circ} 21^{\prime} 23.8^{\prime \prime}$ & a & a & a & a \\
\hline Average & & & $64.94 \pm 5.99$ & $0.51 \pm 0.05$ & $1.94 \pm 0.18$ & $1.21 \pm 0.11$ \\
\hline Maximum & & & $171.17 \pm 15.80$ & $1.27 \pm 0.12$ & $5.19 \pm 0.48$ & $3.28 \pm 0.26$ \\
\hline Minimum & & & $28.28 \pm 2.61$ & $0.21 \pm 0.02$ & $0.38 \pm 0.04$ & $0.42 \pm 0.04$ \\
\hline a Water sa & nples could not & ken & & & & \\
\hline
\end{tabular}




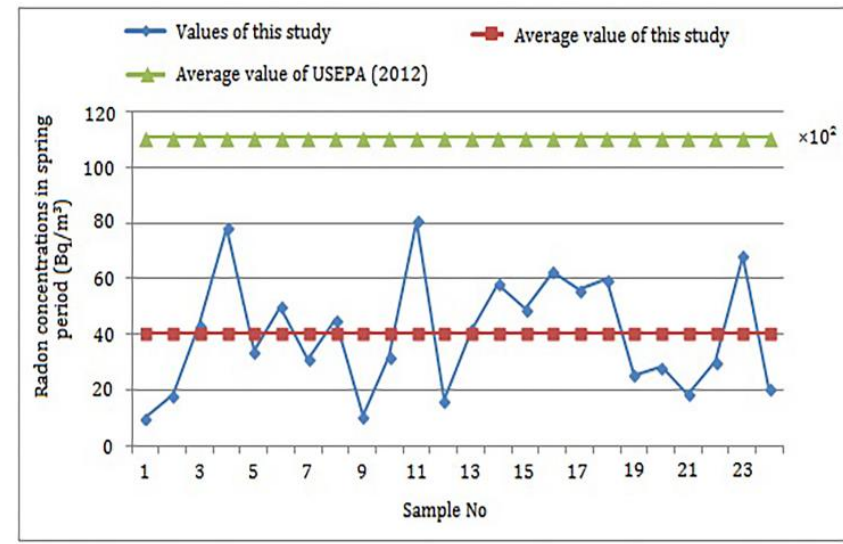

Figure 4. Comparison of radon concentration values in surface water samples of spring period with the value recommended by United States Environmental Protection Agency (USEPA, 2012).

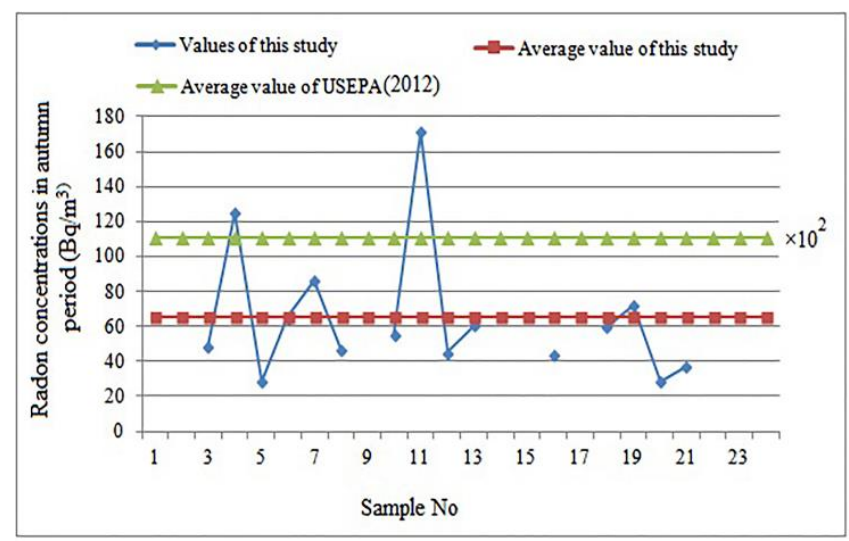

Figure 5. Comparison of radon concentration values in surface water samples of autumn period with the value recommended by United States Environmental Protection Agency (USEPA, 2012).

The highest $C_{R n}, E_{s}, E_{m}$ values in spring water samples were found in the sample Y.S-11. Similarly, the highest $C_{R n}, E_{s}, E_{m}$ and $\mathrm{EC}_{\mathrm{Ra}}$ values in autumn water samples were found in the sample Y.S-11. While the lowest $\mathrm{C}_{\mathrm{Rn}}$ value obtained for water samples belonged to the spring period Y.S-1 sample with $10.00 \pm 0.92$ $\mathrm{Bq} / \mathrm{m}^{3}$, the highest $\mathrm{C}_{\mathrm{Rn}}$ value belonged to the autumn period Y.S-11 sample with $171.17 \pm 15.80 \mathrm{~Bq} / \mathrm{m}^{3}$. While the lowest $\mathrm{E}_{\mathrm{s}}$ value belonged to the spring period YS-9 sample with $(0.08 \pm 0.01) \times 10^{-1} \mathrm{~Bq} / \mathrm{m}^{2} . \mathrm{h}$, the highest $\mathrm{E}_{\mathrm{s}}$ value belonged to the autumn period YS-11 sample with $(1.27 \pm 0.12) \times 10^{-1} \mathrm{~Bq} / \mathrm{m}^{2} . \mathrm{h}$. $E_{m}$ value was calculated as the lowest with $(0.14 \pm 0.01) \times 10^{-3}$ $\mathrm{Bq} / \mathrm{m}^{3}$ at Y.S-1 sample in spring period and the highest with $(5.19 \pm 0.48) \times 10^{-3} \mathrm{~Bq} / \mathrm{m}^{3}$ at Y.S-11 sample in autumn period.

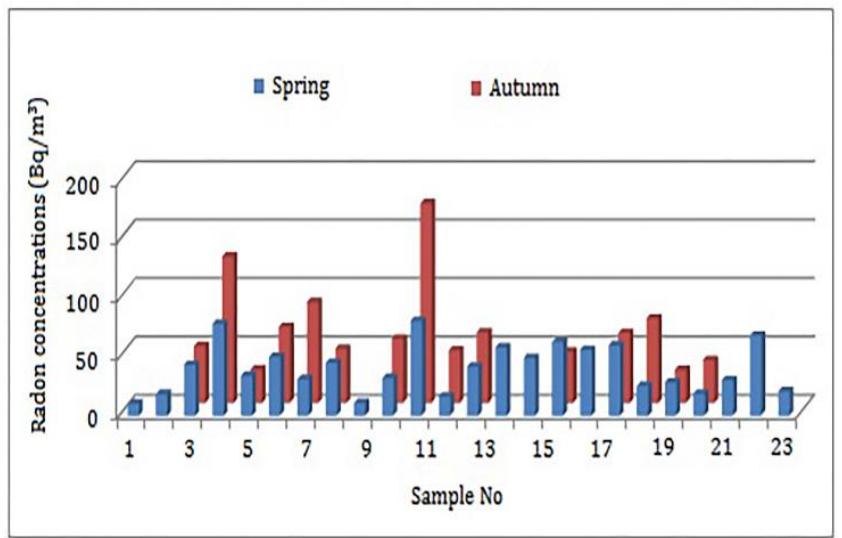

Figure 6. Seasonal variation of radon concentration in samples

Finally, in all water samples, $\mathrm{EC}_{\mathrm{Ra}}$ value was determined to be the lowest with $(0.08 \pm 0.01) \times 10^{-1} \mathrm{~Bq} / \mathrm{m}^{3}$ at Y.S-23 sample in spring period and the highest with $(3.28 \pm 0.26) \times 10^{-1} \mathrm{~Bq} / \mathrm{m}^{3}$ at Y.S-9 sample in autumn period.

Comparison of radon concentration averages obtained for surface water samples in this study with the results obtained in similar studies was given in Table 3. 
Table 3. Comparison of radon concentration averages obtained for water samples in this study with the results obtained in similar studies

\begin{tabular}{|c|c|c|}
\hline Countries & $\begin{array}{l}\text { Concentration of } \\
{ }^{222} \mathrm{Rn}\left(\mathrm{Bq} / \mathrm{m}^{3}\right)\end{array}$ & Reference \\
\hline $\begin{array}{l}\text { England (English } \\
\text { Lake District) }\end{array}$ & $\begin{array}{l}53.7 \pm 8.15- \\
1130.74 \pm 35.92\end{array}$ & $\begin{array}{l}\text { Al-Masri and } \\
\text { Blackburn, } 1999\end{array}$ \\
\hline $\begin{array}{l}\text { Turkey } \\
\text { (Yeşilırmak } \\
\text { River) }\end{array}$ & $280 \pm 40-1080 \pm 300$ & Oner et al., 2009 \\
\hline $\begin{array}{l}\text { Egypt (Manzala } \\
\text { Lake) }\end{array}$ & $(1.720-6.400) \times 10^{3}$ & Yousef et al., 2017 \\
\hline $\begin{array}{l}\text { China (Yunlong } \\
\text { Lake) (average) }\end{array}$ & $2.154 \times 10^{3}$ & Yang et al., 2020 \\
\hline $\begin{array}{l}\text { India (Varahi } \\
\text { River) }\end{array}$ & $(2.07 \pm 0.84) \times 10^{3}$ & $\begin{array}{l}\text { Somashekar and } \\
\text { Ravikumar, } 2010\end{array}$ \\
\hline $\begin{array}{l}\text { India } \\
\text { (Markandeya } \\
\text { River) (average) }\end{array}$ & $(9.30 \pm 1.45) \times 10^{3}$ & $\begin{array}{l}\text { Somashekar and } \\
\text { Ravikumar, } 2010\end{array}$ \\
\hline UNSCEAR & $10 \times 10^{3}$ & UNSCEAR, 2000 \\
\hline USEPA & $11.1 \times 10^{3}$ & USEPA, 2012 \\
\hline EPA & $11 \times 10^{3}$ & EPA, 2000 \\
\hline WHO & $100 \times 10^{3}$ & WHO, 2008 \\
\hline $\begin{array}{l}\text { Turkey (Van } \\
\text { Lake) (average) }\end{array}$ & $\begin{array}{l}40.43 \pm 3.73 \text { (spring) } \\
64.94 \pm 5.99 \text { (autumn) }\end{array}$ & Present Study \\
\hline
\end{tabular}

Baykara and Doğru (2006) collected 14 water samples from the region of the North and East Anatolian Active Faults. By using the passive radon measurement method, they calculated the highest radon concentration, effective radium content and radon diffusion rate in water samples as $3319.3 \mathrm{~Bq} / \mathrm{m}^{3}, 270.5$ $\mathrm{Bq} / \mathrm{m}^{3}$ and $0.470 \mathrm{~Bq} / \mathrm{m}^{2} \cdot \mathrm{h}$, respectively (Baykara and Doğru, 2006).

Bal and Doğru (2013), passively examined radon concentrations in the water samples they received from the radon monitoring stations they established in the Sivrice Fault Zone. As a result of the study, it was seen that the radon concentration values of the water samples taken in autumn and spring varied between $2750 \pm 736 \mathrm{~Bq} / \mathrm{m}^{3}$ and $4624 \pm 1251$ $\mathrm{Bq} / \mathrm{m}^{3}$ with $4464 \pm 1524 \mathrm{~Bq} / \mathrm{m}^{3}$ and $7163 \pm 1941 \mathrm{~Bq} / \mathrm{m}^{3}$, respectively (Bal and Doğru, 2013).

According to the study conducted by Zorer et al., (2013), the radon concentration in lake water around Van Lake varied between $47.80 \mathrm{~Bq} / \mathrm{m}^{3}$ and $354.86 \mathrm{~Bq} / \mathrm{m}^{3}$ (Zorer et al., 2013).

United States Environmental Protection Agency (USEPA) recommends the maximum value of the radon activity level as
11.1 Bq/L (11.1 $\left.\times 10^{3} \mathrm{~Bq} / \mathrm{m}^{3}\right)$ in drinking water (USEPA, 2012). The United Nations Scientific Committee on the Effects of Atomic Radiations (UNSCEAR) has suggested the radon concentration in drinking water must lies between 4 and $40 \mathrm{~Bq} / \mathrm{L}$ (UNSCEAR, 2008).

In present study, the radon concentration values calculated in all samples were lower than $100 \mathrm{~Bq} / \mathrm{L}\left(100 \times 10^{3} \mathrm{~Bq} / \mathrm{m}^{3}\right)$ recommended for drinking water by European Commission (EU, 2001) and World Health Organization (WHO, 2004).

The results obtained in water samples of this study were found to be lower than the results obtained in the above studies and the limit values given for water.

Considering Table 1, Table 2 and Figure 6, when the $C_{R n}, E_{s}, E_{m}$ and $\mathrm{EC}_{\mathrm{Ra}}$ values obtained for the surface water samples taken in spring and autumn were compared seasonally, it was observed that the $C_{R n}, E_{s}, E_{m}$ and $E_{R a}$ values in spring were lower than those from autumn. This may result from the high rainfall in spring and excessive flows from other water sources into the lake.

\section{Conclusions}

In this study, the seasonal variation in the $\mathrm{C}_{\mathrm{Rn}}, \mathrm{E}_{\mathrm{s}}, \mathrm{E}_{\mathrm{m}}$ and $\mathrm{EC}_{\mathrm{Ra}}$ values in the surface water samples taken in spring and autumn from 24 different points of Van Lake was investigated. Measurements were made using the Passive Radon Detector System (RadoSYS system).

At the end of the study, the highest $\mathrm{C}_{\mathrm{Rn}}, \mathrm{E}_{\mathrm{s}}, \mathrm{E}_{\mathrm{m}}$ and $\mathrm{EC}_{\mathrm{Ra}}$ values were calculated for the S-11 point. The Suphan fault line passes through the region where S-11 is located, and this area densely contains volcanic sediments and late Miocene-Quaternary. In a study, Ramola et al. (2006) discovered that the radon level was higher in the areas comprised of granite, quartz-porphyry, schist and phyllite slates, and lower in the areas with sedimentary rocks.

The radon measurement results obtained in this study were found to be lower than the limit values permitted by USEPA (2012), UNSCEAR (2008), EU (2001) and WHO (2004) and results obtained in similar studies.

When the results obtained for the $C_{R n}, E_{s}, E_{m}$ and $E_{R a}$ parameters were compared seasonally, it was seen that the results obtained in the autumn period were higher than those in the spring period.

\section{Acknowledgements}

This study was supported by Scientific Research Projects Coordination Unit of Firat University (FÜBAP), Project number: FF.14.22. The authors thank the Scientific Research Projects Coordination Unit of Firat University. 


\section{References}

Alali, A.E., Al-Shboul, K.F., Yaseen, Q.B., and Alaroud, A., 2019. Assessment of radon concentrations and exposure doses in dwellings surrounding a high capacity gas turbine power station using passive measurements and dispersion modeling. Journal of Environmental Radioactivity, 196, 9-14.

Alkan, H., and Göksel, S.A., 1975. Türkiye Kaplıca ve Maden Sularının Doğal Radyoaktiviteleri, Sularda Radon Tayini, T. B. T. A. K. 5. Bilim Kongresi, Ankara, 229-241.

Yaramıș, B., 1985. Nükleer Fizik. 1, İ.T.Ü. Fen-Edebiyat Fakültesi, İstanbul.

Al-Masri, M.S., and Blackburn, R., 1999. Radon-222 and related activities in surface waters of the English Lake District. Applied Radiation and Isotopes, 50, 1137-1143.

Bal, S.Ş., and Doğru, M., 2013. Su ve toprak örneklerinde radon gazı yayılımının mevsimsel değişiminin incelenmesi. BEÜ Fen Bilimlerı Dergisi, 2(2), 192-196.

Baykara, O., and Doğru, M., 2006. Measurements of radon and uranium concentration in water and soil samples from East Anatolian Active Fault Systems (Turkey), Radiat. Meas., 41, 362-367.

Bohus-Saja, L., Gomez, J., Capote, T., Greaves, E.D., Herrera, O., Salazar, V., and Smith, A.J., 1997. Environ. Radioact., 35, 305-312.

Büyükuslu, H., Özdemir, F.B., Öge, T.Ö., and Gökce, H., 2018. Indoor and tap water radon $\left({ }^{222} \mathrm{Rn}\right)$ concentration measurements at Giresun University campus areas. Applied Radiation and Isotopes, 141, 95-100.

EPA (Environmental Protection Agency), 2000. Final Rule for NonRadon Radionuclides in Tap Water, Technical Fact Sheet, EPA, 815-F00-013, 2000.

EU (European Commission), 2001. Commission recommendation of 20th December 2001 on the protection of the public against exposure to radon in drinking water suplies (2001/928/Euratom). Off. J. Eur. Comm. L:344/85.

Ilani, S., Minster, T., Kronfeld, J., and Even, 0., 2006. The source of anomalous radioactivity in the springs bordering the Sea of Galilee, Israel. Journal of Environmental Radioactivity, 85, 137-146.

Jönsson, G., Baixeras, C., Devantier, R., Enge, W., Font, L.L., Freyer, K., Ghose, R., and Treutler, H.C., 1999. Soil radon levels measurements with SSNTD's and the soil radium content, Radiat. Meas., 31, 291-294.

Kayakökü, H., Karatepe, Ş., and Doğru, M., 2016. Measurements of radioactivity and dose assessments in some building materials in Bitlis, Turkey Applied Radiation and Isotopes, 115, 172-179.

Kayakökü, H., and Doğru, M., 2019. "Seasonal Radon Measurements in Surface Water Samples from Van Lake, Turkey" XII. International Conference on Nuclear Structure Properties (NSP 2019), Poster Presentation, 11-13 September 2019, Bitlis, Turkey.

Oner, F., Yalim, H.A., Akkurt, A., and Orbay, M., 2009. The measurements of radon concentrations in drinking water and the Yeşilırmak River water in the area of Amasya in Turkey Protection Dosimetry, 133(4), 223-226.

Ramola, R.C., Choubey, V.M., Prasad, Y., Prasad, G., and Bartarya, S.K.,
2006. Variation in radon concentration and terrestrial gamma radiation dose rates in relation to the lithology in southern part of Kumaon Himalaya, India. Radiat. Meas., 41: 714-720.

Sannappa, J., Chandrashekara, M.S., and Paramesh, L., 2006. Spatial distribution of radon and thoron concentrations indoors and their concentrations in different rooms of buildings. Indoor and Built Environment, 15(3), 283-288.

Shashikumar, T.S., Chandrashekara, M.S., Nagaiah, N., and Paramesh, L., 2009. Variations of radon and thoron concentrations in different types of dwellings in Mysore city, India. Radiation Protection Dosimetry, 133(1), 44-49.

Somashekar, R.K., and Ravikumar, P., 2010. Radon concentration in groundwater of Varahi and Markandeya river basins, Karnataka State, India. J Radioanal Nucl Chem., 285, 343-351.

Tokonami, S., Takahashi, H., Kobayashi, Y., Zhuo, W., and Hulber, E., 2005. Up-to-date radon-thoron discriminative detector for a large scale survey, Rev. Sci. Instrum. 76, 113-505.

Turhan, Ş., Demir, K., and Karataşli, M., 2018. Radiological evaluation of the use of clay brick and pumice brick as a structural building material. Applied Radiation and Isotopes, 141, 95-100.

UNSCEAR (United Nations Scientific Committee on the Effects of Atomic Radiations), 2000. Sources and effects of ionizing radiation. Report to the General Assembly with scientific annexes (pp. 97-105). United Nations, New York, Annexure B.

UNSCEAR (United Nations Scientific Committee on the Effects of Atomic Radiations), 2008. Report to General Assembly with Scientific annexes. United Nations Sales Publications. United Nations, New York.

USEPA (United States Environmental Protection Agency), 2012. Edition of the Drinking Water Standards and Health Advisories. EPA 822-S-12-001 Office of Water U.S., Environmental Protection Agency, Washington, DC.

WHO (World Health Organization), 2004. Guidelines for DrinkingWater Quality. Vol. 1. Recommendations, third ed., Geneva.

WHO (World Health Organization), 2008. Guidelines for DrinkingWater Quality. Vol. 1. Recommendations, third ed., Incorporating the First and Second Addenda, Geneva.

Varol, S., 2011. Gross alpha and beta radioactivity in groundwaters. Journal of Engineering Science and Design, 1(3), 101-106.

Yang, J., Yu, Z., Yi, P., Frape, K.S., Gong, M., and Zhang, Y., 2020. Evaluation of surface water and groundwater interactions in the upstream of Kui River and Yunlong Lake, Xuzhou, China. Journal of Hydrology, 583, 124549.

Yaramıș, B., 1985. Nükleer Fizik. 1, İ.T.Ü. Fen-Edebiyat Fakültesi, İstanbul.

Yousef, H.A., El- Farrash, A.H., and Magdy, A., 2017. Radon Levels in Surface Water Samples from Manzala Lake East Nile Delta, Egypt using Nuclear Track Detectors. Journal of Nuclear and Particle Physics, 7(2), 36-42.

Zorer, Ö.S., Şahan, T., Ceylan , H., Doğru, M., and Şahin, S., 2013. ${ }^{238 U}$ and ${ }^{222} \mathrm{Rn}$ activity concentrations and total radioactivity levels in lake waters, J. Radioanal. Nucl. Chem., 295, 1837-1843. 